\title{
Turbulence in the Diffuse Interstellar Medium
}

\author{
Edith Falgarone ${ }^{1}$, Benjamin Godard ${ }^{2}$ and Pierre Hily-Blant ${ }^{3}$ \\ ${ }^{1}$ LERMA-LRA, Ecole Normale Supérieure \& Observatoire de Paris, Paris, France \\ email: edith.falgarone@ens.fr \\ ${ }^{2} \mathrm{CAB} / \mathrm{CSIC}$, Madrid, Spain, email: bgodard@cab.inta-csic.es \\ ${ }^{3}$ IPAG, Grenoble, France, email: pierre.hily-blant@obs.ujf-grenoble.fr
}

\begin{abstract}
The diffuse interstellar medium (ISM) hosts the first steps of interstellar chemistry and the seeds of dense structures. Since its turbulent pressure by far exceeds its thermal pressure, turbulence must play a prominent role in its evolution. Fed at galactic scales, turbulent energy cascades down to the dissipation scales, but as in both laboratory and atmospheric turbulence, it does so in an intermittent way : only a tiny fraction of the small-scales is fed by the turbulent cascade, so that dissipation occurs in bursts. In diffuse molecular clouds, where they can be observed, the signatures of intermittency are: (1) the non-Gaussian statistics of velocity increments, and (2) the existence of coherent structures of intense velocity-shear that appear to channel the large-scale turbulent energy down to milliparsec scales. Attempts at modelling the warm chemistry triggered in the diffuse ISM by bursts of turbulent dissipation are promising : in this framework, the so far unexplained molecular richness observed in this medium is naturally understood, in particular its $\mathrm{CH}^{+}, \mathrm{HCO}^{+}$and $\mathrm{CO}$ abundances. Turbulent dissipation is also likely at the origin of the $\mathrm{H}_{2}$ rotational line emission of the diffuse ISM and of a significant fraction of its [C II] emission.
\end{abstract}

Keywords. astrochemistry, turbulence, ISM: clouds, ISM: general, ISM : evolution, ISM: kinematics and dynamics, ISM: molecules, ISM: structure, submillimeter

\section{Introduction: the diffuse neutral interstellar medium}

The definition of the diffuse neutral interstellar medium (ISM) has evolved continuously since its fortuitous discovery by Hartmann in 1904, depending on the observational techniques used to probe it. It is now generally agreed that it is a low density medium $\left(n_{\mathrm{H}}<\right.$ a few $\left.10^{3} \mathrm{~cm}^{-3}\right)$ weakly shielded from the interstellar radiation field (ISRF) $\left(A_{V}<\right.$ a few mag), therefore comprising the diffuse atomic, diffuse molecular and translucent media, following the terminology of Snow \& McCall (2006). An even broader definition of the diffuse ISM is adopted in this paper. It comprises the above plus the edges of molecular clouds as traced by their ${ }^{12} \mathrm{CO}(J=1-0)$ emission, i.e. all the gas that is not in dense cores and/or star forming regions. Equivalently, the diffuse ISM can be considered to be all material with hydrogen column density less than a few $\sim 10^{21} \mathrm{~cm}^{-2}$ at the parsec scale. Such a broad definition is motivated by the shapes of the probability distribution functions $(p d f)$ of the column density (inferred from dust extinction) of nearby molecular clouds : they have a log-normal part from $A_{V} \sim 0.5 \mathrm{mag}$, up to $A_{V} \sim$ a few magnitude, and power-law tails at higher column densities, characteristics of dense cores and filaments, present only in clouds actively forming stars (Kainulainen et al., 2009). The log-normal contribution comes from the diffuse ISM of these molecular clouds. The diffuse ISM, at large, may therefore be seen as all the gas that does not belong to smallscale self-gravitating structures such as dense cores, dense filaments and star-forming 


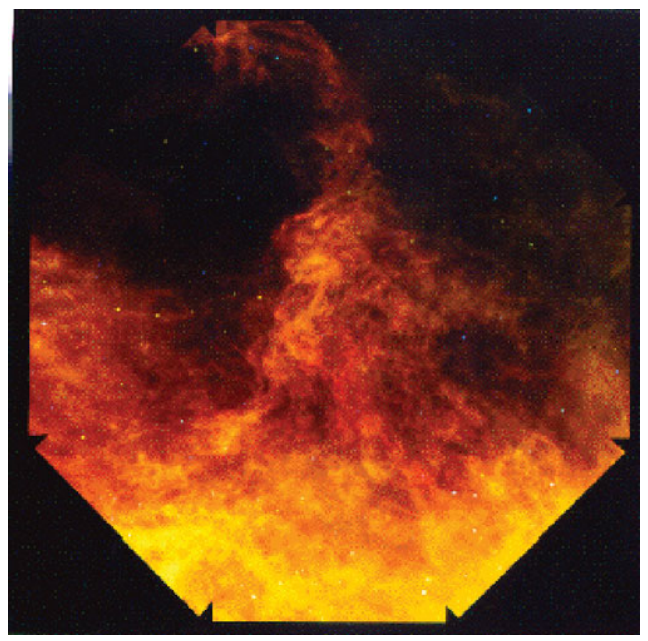

Figure 1. IRIS $100 \mu \mathrm{m}$ image of the dust thermal emission of the North Celestial Loop (Miville-Deschênes \& Lagache 2005) rising $\sim 100 \mathrm{pc}$ above the Galactic plane visible at the bottom. The size of the map is about $30^{\circ}$. The $40^{\prime} \times 30^{\prime}$ field of the Polaris Flare discussed in Sect. 4 is located close to the center of the image.

regions. Note that the diffuse medium, as defined above, comprises the bulk of the mass of molecular clouds, at the 30 pc scale (e.g. Goldsmith et al., 2008).

\subsection{Thermal and non-thermal pressures}

The shape of the cooling function dominated by [C II] line emission makes the diffuse ISM thermally bistable for the pressure and metallicity conditions prevailing in the solar neighbourhood (Field et al., 1969). The balance between heating and radiative cooling processes generates two stable phases at very different temperatures and densities, $T \sim 100 \mathrm{~K}$ and $n_{\mathrm{H}} \sim 30 \mathrm{~cm}^{-3}$ for the cold neutral medium (CNM), $T \sim 8000 \mathrm{~K}$ and $n_{\mathrm{H}} \sim 0.3 \mathrm{~cm}^{-3}$ for the warm neutral medium (WNM). However, a broad range of kinetic temperatures, pervading the thermally unstable range, is usually inferred from UV absorption lines sampling the diffuse ISM (e.g. Fitzpatrick \& Spitzer, 1997), and recent sensitive HI observations also probe temperatures in this range (Begum et al., 2010; Heiles \& Troland, 2003). The latter authors infer that about half the WNM is at temperatures in the range $500-5000 \mathrm{~K}$, thus evolving between the two stable phases.

The diffuse ISM is now modeled as a turbulent medium, evolving far out of thermal pressure equilibrium, as illustrated by the dust thermal emission of the North Celestial Loop (Fig. 1). Whether it is cooling (and condensing) WNM, destabilized in colliding flows, as in the numerical simulations of Audit \& Hennebelle (2005), or CNM transiently heated by turbulent dissipation, in the framework discussed below, is presently an open issue.

Because it sits in the gravitational potential of the Galaxy, the diffuse ISM has to be supported by a pressure that is about 10 times its thermal pressure, $P_{t o t}=3 \times 10^{-12}$ dynes $\mathrm{cm}^{-2}$, or $P_{t o t} / k \sim 2 \times 10^{4} \mathrm{~cm}^{-3} \mathrm{~K}$ (Cox 2005). The non-thermal contributions to the total pressure are due to supersonic turbulence and magnetic fields, in rough equipartition, as shown by measurements of magnetic field intensity (Crutcher et al., 2010) and HI linewidths (Haud \& Kalberla, 2007). The distribution of thermal pressures in the solar neighbourhood, inferred from [CI] fine-structure absorption lines towards nearby stars (Jenkins \& Tripp, 2011) peaks at about $P_{t h} / k \sim 3 \times 10^{3} \mathrm{~cm}^{-3} \mathrm{~K}$, with large fluctuations, up to a few $10^{4} \mathrm{~cm}^{-3} \mathrm{~K}$. It is interesting that the total non-thermal pressure in the Galactic plane is of the same order as the largest thermal pressure observed, suggesting that the non-thermal energy eventually and occasionally degrades into thermal energy.

These large fluctuations of thermal pressure are a sign of a complex dynamic evolution that may provide the missing important clues to the so-called "outstanding mysteries" 
raised by the observations of diffuse molecular gas (see the review of Snow \& McCall, 2006): the ubiquitous small scale structure, down to AU-scales, and its remarkable molecular richness found in this hostile medium weakly protected from UV radiation (e.g. Liszt \& Lucas, 1998).

\subsection{Two outstanding mysteries}

A resilient major puzzle is the existence in the diffuse ISM of molecular species that have a formation endothermicity far above the available thermal energy: their large observed abundances cannot be reproduced by chemistry models driven by UV photons and cosmic-rays. This has been known for 70 years in the case of $\mathrm{CH}^{+}$that not only forms through the highly endothermic reaction $\mathrm{C}^{+}+\mathrm{H}_{2}(\Delta E / k=4940 \mathrm{~K})$ but also is rapidly destroyed by collisions with $\mathrm{H}_{2}$. Until recently, observation of its electronic transition in absorption against nearby stars was the only means of investigating this species in the diffuse gas (Crane et al., 1995; Gredel et al., 1997; Weselak et al., 2008) because the frequency of its ground-state rotational transition $(835 \mathrm{GHz})$ falls in an opaque domain of the atmosphere. Hence, only the solar neigbourhood had been sampled. The Herschel/HIFI observations of the $\mathrm{CH}^{+}(1-0)$ and ${ }^{13} \mathrm{CH}^{+}$(1-0) transitions in absorption against the dust continuum emission of remote sources (Falgarone et al., 2010a,b) now extend the puzzle raised by the large abundances of $\mathrm{CH}^{+}$in the local diffuse medium to the whole Galactic disk (Fig. 2). Similar large $\mathrm{CH}^{+}$abundances are found over a broad range of total hydrogen column densities with a similar large scatter by a factor of 10 (or more) about the average value. The puzzle is even sharper now that $\mathrm{SH}^{+}$is also observed to be abundant in the diffuse ISM : this species has a larger formation endothermicity, via $\mathrm{S}^{+}+\mathrm{H}_{2}$, than that of $\mathrm{CH}^{+}, \Delta E / k=9860 \mathrm{~K}$ (Menten et al., 2011; Godard et al., 2011).

Another outstanding and more recent puzzle is the origin of the $\mathrm{H}_{2}$ pure rotational line emission of the diffuse ISM. Its discovery dates back to the ISO-SWS detection of the four lowest rotational transitions across a large pathlength of diffuse gas in the Galactic plane, $N_{\mathrm{H}} \sim 10^{22} \mathrm{~cm}^{-2}$, equivalent to $16 \mathrm{mag}$ (Falgarone et al., 2005). Given the high energy of the upper level of these transitions, $510 \mathrm{~K}$ for the $\mathrm{S}(0)$ line, up to $2540 \mathrm{~K}$ for the $\mathrm{S}(3)$ line, and the absence of intense UV radiation field able to excite these levels by fluorescence, a non-thermal excitation of some kind is required. This Galactic emission was modelled by advocating heating of the gas by turbulent dissipation. The dissipation regions, distributed along the line of sight, were either hundreds of low velocity magnetohydrodynamical (MHD) shocks ( 8 to $12 \mathrm{~km} \mathrm{~s}^{-1}$ ) or thousands tiny regions of intense velocity-shears, heating the gas by ion-neutral friction and/or viscous dissipation. The interesting new result of these models was that a fraction as small as a few per cent of warm gas, heated by dissipation of mechanical energy, was sufficient to reproduce the observed line intensities, and their line ratios. New detections of these lines have been performed by Spitzer/IRS, in the diffuse medium sampled in the fields of supernova remnants (Hewitt et al., 2009), in a translucent field of the Taurus molecular complex (Goldsmith et al., 2010), in the Polaris Flare (Falgarone et al., in prep.) and in diffuse clouds of the Chamaeleon complex and LDN 1780 (Ingalls et al., in prep.): in these studies, UV excitation cannot explain the line intensities and non-thermal excitation, likely fed by turbulence, is required.

\section{A few words on turbulence}

The definition of turbulence is built on experiment. Turbulence is an instability of laminar flows that develops as soon as the inertial $\mathbf{v} \cdot \nabla \mathbf{v}$ forces greatly exceed the viscous 

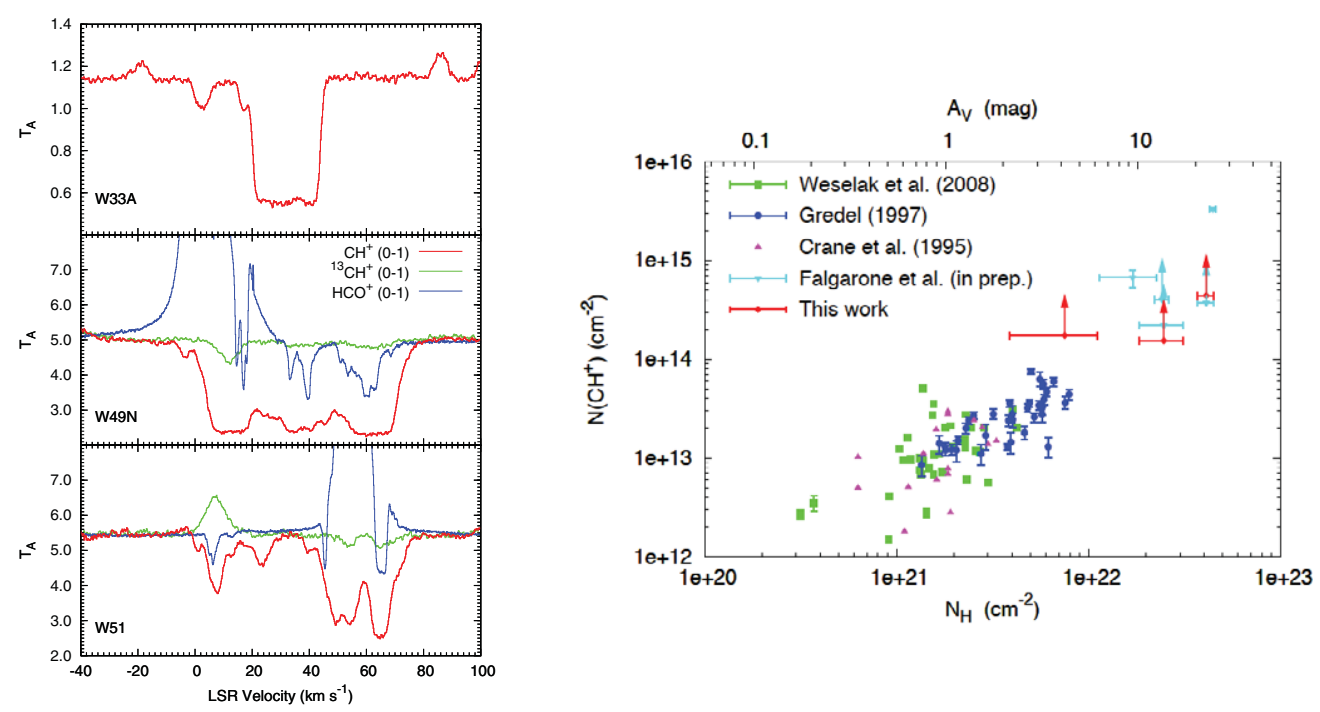

Figure 2. Left: $\mathrm{CH}^{+}(1-0),{ }^{13} \mathrm{CH}^{+}(1-0)$ and $\mathrm{HCO}^{+}(1-0)$ spectra obtained with the Herschel/HIFI instrument in the direction of three remote star forming regions. Note the saturated shape of the $\mathrm{CH}^{+}$lines over large velocity intervals and the difference between the lineprofiles of $\mathrm{HCO}^{+}$and $\mathrm{CH}^{+}$. Right: $\mathrm{CH}^{+}$column density vs. total hydrogen column density on sight-lines towards nearby stars and far-IR star-forming regions. All data sets are consistent with a linear increase of $\mathrm{CH}^{+}$with the total hydrogen column density, with however a large scatter, providing abundances in the range $N\left(\mathrm{CH}^{+}\right) / N_{\mathrm{H}}=10^{-9}-2 \times 10^{-8}$.

$\nu \Delta \mathbf{v}$ forces ( $\nu$ is the kinematic viscosity) i.e. when the Reynolds number $R e=l v_{l} / \nu$, at a scale $l$ of characteristic velocity $v_{l}$, exceeds a few hundreds. This instability at scale $l$ is at the origin of an energy transfer to smaller scales, that eventually become unstable too and transfer their kinetic energy to still smaller scales, etc... This is the turbulent cascade that develops between the integral scale, $L$, at which energy is injected, and the dissipation scale $l_{D}$, close to the particle mean-free-path, where energy is dissipated into heat due to the particle viscosity. The timescale for the growth of this instability is of the order of the turnover time $\tau_{l}=l / v_{l}$ at each length scale $l$. Kolmogorov (1941) predicted the self-similar behavior of the velocity field in incompressible turbulence by postulating a dissipationless cascade characterized by a transfer rate of kinetic energy independent of scale, $\epsilon \propto v_{l}^{2} / \tau_{l}=v_{l}^{3} / l$, hence the well-known scaling $v_{l} \propto l^{1 / 3}$. It is easy to demonstrate that this assumption leads to an energy spectrum $E(k) \propto k^{-5 / 3}$ known as the Kolmogorov spectrum. $E(k)$ has the dimension of a kinetic energy per unit mass and unit wavenumber because the average specific kinetic energy at scale $l=2 \pi / k$ is $\left\langle v_{l}^{2}\right\rangle=\int_{k}^{\infty} E\left(k^{\prime}\right) d k^{\prime}$. In Kolmogorov turbulence, the turnover timescale $\tau_{l}$ therefore decreases towards small-scales while the velocity gradient $v_{l} / l \propto l^{-2 / 3}$ slowly increases.

For half a century now, turbulence has been recognized to be intermittent i.e. the smaller the scale, the larger the spatio-temporal velocity fluctuations, relative to their average value (Landau \& Lifchitz 1959, Kolmogorov 1962). Turbulent energy is not evenly distributed in space and time by the turbulent cascade : at each step of the cascade, the active sub-scales do not fill space so that the subset of space on which the active scales are distributed has a multifractal geometry (see the review of Anselmet et al., 2001 and the book of Frisch, 1995). The statistical properties of the velocity fluctuations have been widely studied experimentally in laboratory and atmospheric flows : in all cases, 

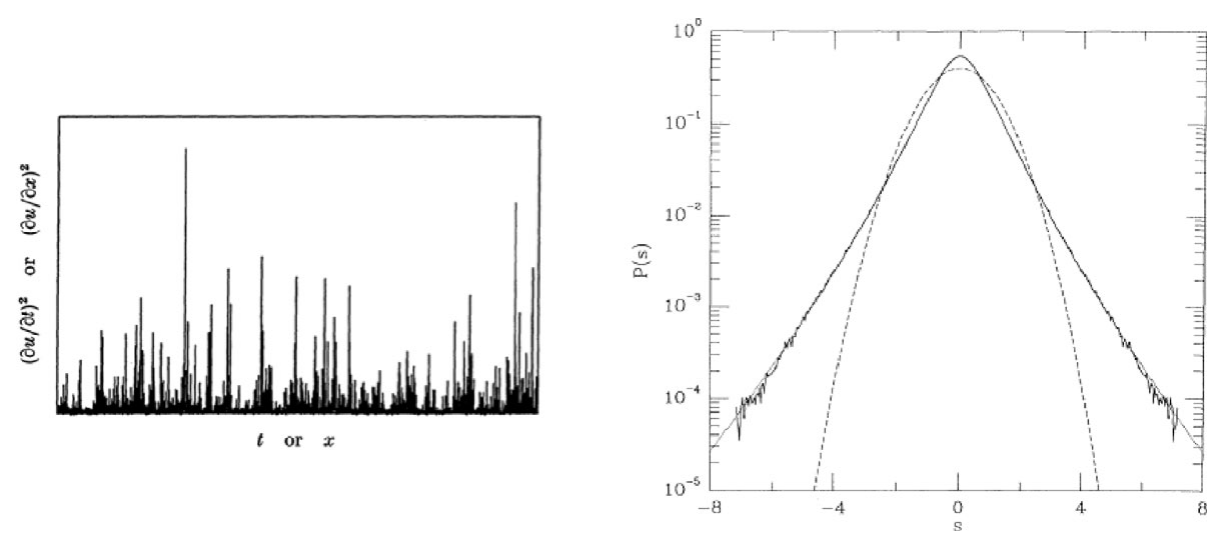

Figure 3. Left: A record of the square of the velocity derivative (with respect to time or space) measured in the turbulent wake of a cylinder (Sreenivasan et al., 1989). This signal can be viewed as the rate of dissipation of turbulent kinetic energy. The rare largest excursions are more frequent than they would be if the signal were Gaussian. Right: Non-Gaussian probability distribution of the longitudinal velocity differences $\delta v_{x}(s)=v_{x}(x+s)-v_{x}(x)$ measured in such a flow over a lag $s$ much smaller than the integral scale. The dashed line shows a Gaussian probability distribution with same dispersion.

the statistics of velocity derivative and increments signals are found to be non-Gaussian, with large departures from the average more frequent than for a Gaussian distribution (Fig. 3). The $p d f$ of the turbulent velocity field, in turn, remains Gaussian. Moreover, the departure of the velocity increments $p d f \mathrm{~s}$ from a Gaussian distribution increases as the lag over which the increments are measured decreases. All the functions of the velocity involving a spatial derivative have therefore non-Gaussian $p d f \mathrm{~s}$ : the velocity gradients $\left(\partial_{i} v_{i}\right)$ and shears $\left(\partial_{j} v_{i}\right)$ and, accordingly, the rate-of-strain $S_{i j}=\partial_{j} v_{i}+\partial_{i} v_{j}$ and the dissipation rate $\epsilon_{D}=\frac{\nu}{2} \Sigma_{i j}\left(\partial_{j} v_{i}+\partial_{i} v_{j}\right)^{2}$, with non-Gaussian wings more pronounced at small scale.

The quantitative signature of intermittency appears in the behavior of the high-order structure functions of the longitudinal velocity field measured over a lag $s,\left\langle\left[\delta v_{x}(s)\right]^{p}\right\rangle \propto$ $s^{\zeta_{p}}$. This relation is statistical, not deterministic, and the brackets hold for an average over an "appropriate" volume with respect to $s^{3}$. The anomalous scaling of the exponents $\zeta_{p} \neq$ $p / 3$ characterizes the degree of intermittency and provides the multifractal dimension of the most singular structures, i.e. that of the subset of space where the smallest active regions, and turbulent dissipation, are distributed (She \& Levêque, 1994; Anselmet et al., 2001). In incompressible turbulence, the so-called active small scales are those of largest vorticity or velocity shear.

Another essential facet of turbulent intermittency is that these active small scales are not randomly distributed in space but are organized into coherent structures - the sinews of turbulence, as qualified by Moffatt et al. (1994). In incompressible turbulence, these structures tend to be filamentary as shown in Fig. 4, while those of highest rate-of-strain and dissipation are rather in the form of sheets or ribbons, following the analysis of numerical simulations by Moisy \& Jiménez (2004). These structures are remarkable in the sense that they are both large scales i.e. their length is comparable to the integral scale of turbulence, and small scale structures i.e. they have substructure down to the dissipation scale. The coupling between large scales and small scales operated by turbulence is repeatedly found in simulations. Mininni et al. (2006) find that small-scale intermittency is more pronounced in turbulent fields where the large-scale shear is larger, and 


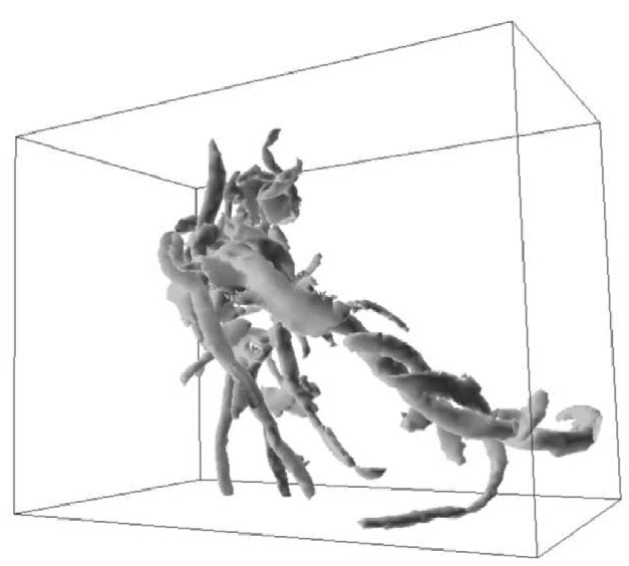

Figure 4. Structure of most intense vorticity found in numerical simulations of 3-dimensional incompressible hydrodynamical turbulence. The pattern shown, consisting of a large tube surrounded by smaller ones wrapped around it, is frequently found (from Moisy \& Jiménez 2004). In magneto-hydrodynamical (MHD) turbulence, structures in which turbulent dissipation is concentrated appear to be more sheet-like (Politano \& Pouquet, 1995).

Federrath et al. (2010) show that solenoidal (incompressible) forcing is able to provide a compressible turbulent flow with the statistical signatures of incompressible turbulence.

\section{Questions raised by diffuse ISM turbulence}

The nature and properties of turbulence in the diffuse ISM are still a highly debated and controversial issue in spite of dedicated observational and numerical efforts. This is due in part to the huge range of scales separating those of the energy injection (at the Galaxy scale and even beyond when infall is taken into account, see de Avillez \& Breitschtweirdt, 2005) from those where it is dissipated, presumably below the milliparsec scale. It is also due to the fact that the turbulence is compressible, magnetized and multiphase. Unraveling the properties of interstellar turbulence is essential though because turbulence and magnetic fields are the main contributions to the pressure of the diffuse ISM (see Sect. 1) and the main support of molecular clouds against their self-gravity. Turbulent dissipation is therefore a key process among those leading to the formation of molecular clouds, star formation, and therefore galaxy evolution. (see the reviews of Elmegreen \& Scalo, 2004 and Scalo \& Elmegreen, 2004).

In the ISM, turbulence is observed to be highly supersonic with respect to the cold gas. Yet, one of the most impressive energy spectrum of turbulence in the diffuse ISM covering more than two orders of magnitude in scales (Fig 5, from Miville-Deschênes et al., 2003) is consistent with the Kolmogorov spectrum of incompressible turbulence, which does not necessarily imply that ISM turbulence is incompressible (see Sect. 2). Interestingly, the energy spectrum of the HI emission has the same slope, $\beta=11 / 3$, over the broad length-scale domain $(0.1-20 \mathrm{pc})$, while that of molecular clouds traced in $\mathrm{CO}(1-0)$ or thermal dust emission is clearly smaller, with a steady increase from $\beta \sim 2$ when measured at the $10-20$ pc scale to $\beta \sim 3.3$ at scales $<1$ pc (see references in the review of Falgarone et al., 2004). Another quantity, the turbulent energy transfer rate, $\epsilon_{l}=\frac{1}{2} \rho v_{l}^{3} / l$, seems to link turbulence in the diffuse atomic medium and that of diffuse molecular gas traced by ${ }^{12} \mathrm{CO}(1-0)$ emission because it has the same value $\epsilon \sim 2 \times 10^{-25}$ $\operatorname{erg} \mathrm{cm}^{-3} \mathrm{~s}^{-1}$ in these two components of the local ISM (see Table II, Falgarone et al., 2004).

Supersonic turbulence is expected to rapidly dissipate in shocks within a cloud crossing time, $\approx 1 \mathrm{Myr}$, for diffuse clouds of a few pc and internal velocity dispersion of a few $\mathrm{km} \mathrm{s}^{-1}$. Numerical experiments show that magnetic fields do not significantly slow the dissipation (MacLow et al., 1998). Whether turbulent dissipation occurs primarily 


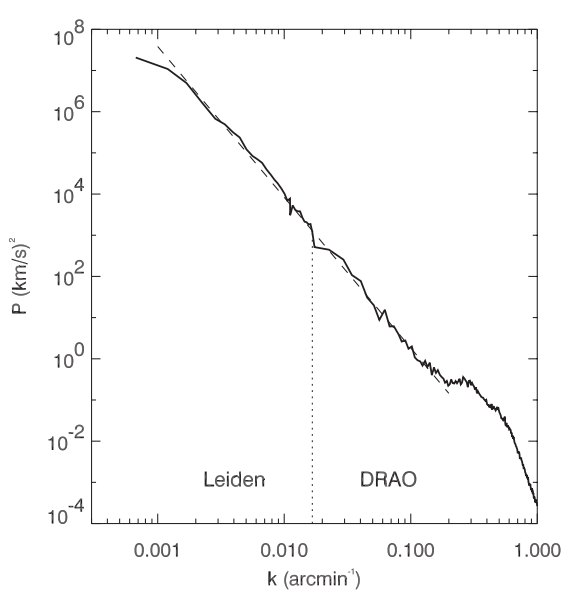

Figure 5. Power spectrum of the velocity field measured with HI emission line profiles in a nearby field at high galactic latitude (Miville-Deschênes et al., 2003). Data obtained with the DRAO interferometer and the Leiden/Dwingeloo survey are merged to provide an unprecedented range of two orders of magnitude in the scale size sampled. The dashed line shows the Kolmogorov spectrum of incompressible turbulence

in compressible (curl-free) or in solenoidal (divergence-free) modes is still an open issue that has important bearings on the subsequent gas evolution and condensation. In their hydrodynamical simulations of mildly compressible turbulence, Porter et al. (2002) show that the compressible component of the velocity field is weaker than its solenoidal counterpart by a factor of $\sim 6$, independent of the nature of the driving process (compressible or solenoidal). Vestuto et al. (2003) find that the energy fraction in the solenoidal modes is dominant and increases with the magnetic field intensity in compressible MHD turbulence. These numerical experiments are still far from approaching conditions in the ISM, but they suggest that, even in the compressible (supersonic) case, turbulent dissipation may occur primarily in solenoidal modes, i.e. primarily velocity-shears, without direct gas compression.

\section{Intermittency of turbulence in the diffuse ISM}

\subsection{Parsec-scale coherent structures of intense velocity-shear}

Identifying regions of intermittency in interstellar turbulence is challenging for several independent reasons. Firstly, these regions are non-space filling and correspond to rare events in time and space (see Fig. 3): their finding requires the analysis of large homogeneous statistical samples of the velocity field. This means observations at high spectral and spatial resolution of large regions of diffuse material. Secondly, observations do not provide the full velocity field but only its line-of-sight (los) projection provided by the Doppler-shift of a molecular line, so statistical analysis similar to those performed in laboratory flows or in the solar wind are not possible. However, measurements of the spectrum of molecular lines with a frequency resolution finer than the sound speed in cold $\mathrm{H}_{2}$ provide information on the velocity field. The ${ }^{12} \mathrm{CO}(1-0)$ line has turned out to be a most useful tool for this search, for reasons that will hopefully become clear to the reader by the end of this paper. Thirdly, only spatial variations of the los velocity in the plane-of-the-sky ( $p o s$ ) are provided by observations. Therefore, the velocity variations are by essence cross-variations $\partial_{j} v_{i}$. Finally, the line emission being integrated along a los, the velocity information at a given position is the full line profile and its moments, the first moment being the line centroid velocity. The statistics of the velocity increments are built through those of the line centroid velocity increments (CVI) measured between two positions separated by a lag $l$ in the pos. The CVIs have been shown by Lis et al. 


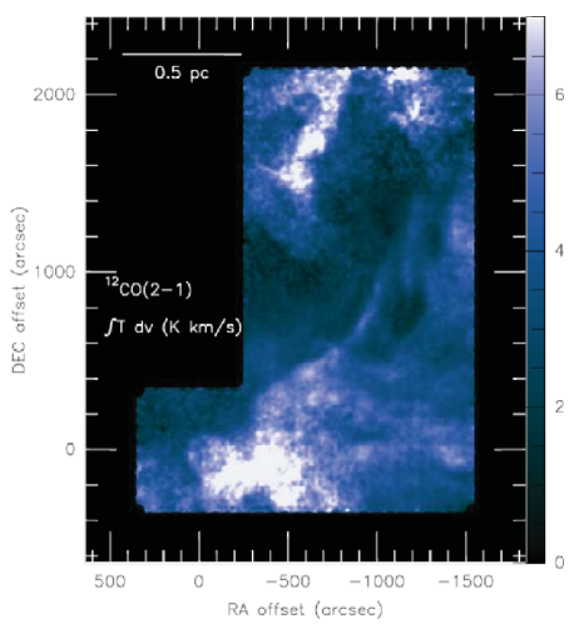

Figure 6. Map of the ${ }^{12} \mathrm{CO}(2-1)$ integrated intensity (in $\mathrm{K} \mathrm{km} \mathrm{s}^{-1}$ ) over a $40^{\prime} \times 30^{\prime}$ area located close to the center of the Polaris Flare field shown in Fig 1. The number of independent spectra is $\sim 10^{5}$. The data have been obtained with the IRAM-30m/HERA mosaic of receivers, operating in the On-The-Fly and frequency-switching mode. The spatial resolution is 11 arcsec or $8 \mathrm{mpc}$ at the distance of the field, $150 \mathrm{pc}$.
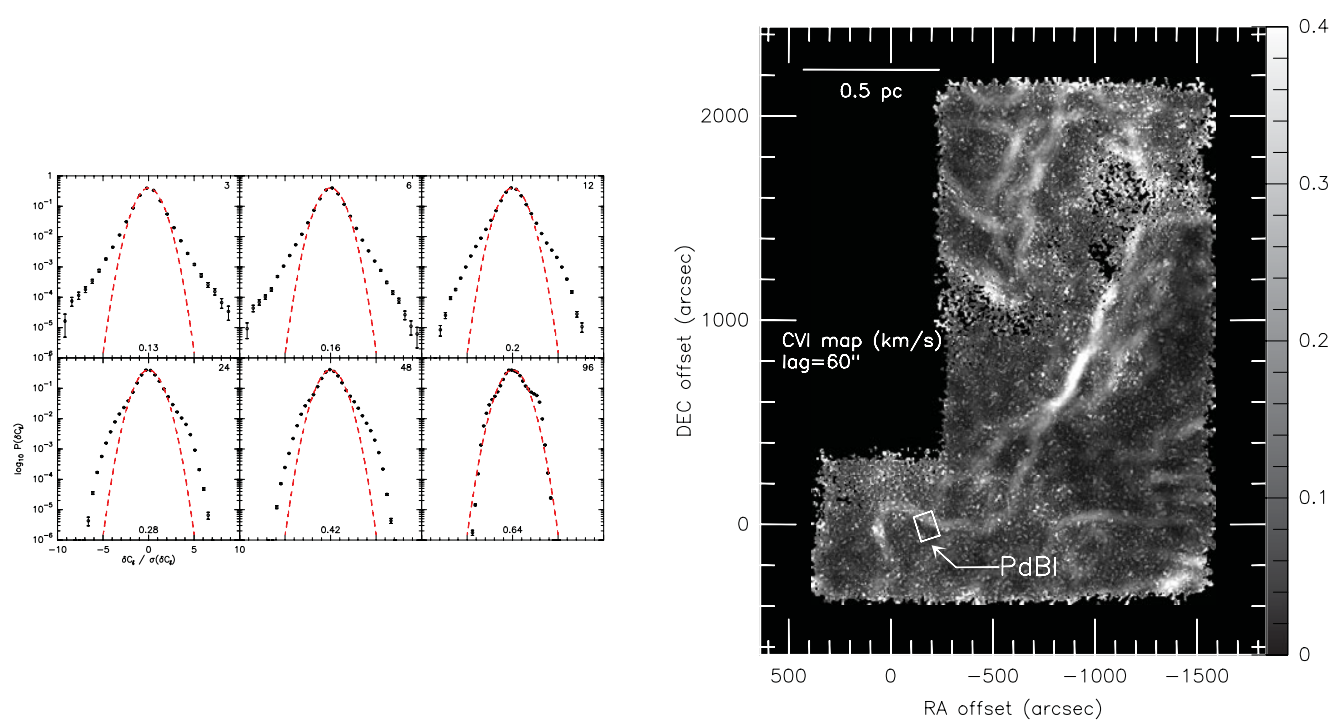

Figure 7. Left: Normalized $p d f \mathrm{~s}$ of line centroid velocity increments (CVI) measured over variable lags, expressed in units of 15 arcsec (upper right corners), and computed within the field of Fig. 6. The Gaussians of same dispersion $\sigma\left(\delta C_{l}\right)$ (given in $\mathrm{km} \mathrm{s}^{-1}$ at the bottom of each panel) are also drawn. The non-Gaussian wings of the $p d f \mathrm{~s}$ increase as the lag decreases. Note that a probability of $10^{-5}$ is reached in the most extreme bins. Right: In the same field, locus of the positions populating the non-Gaussian wings of the $p d f$ for a lag of 60 arcsec. The wedge is in $\mathrm{km} \mathrm{s}^{-1}$. The rectangle is the area observed with the IRAM-PdBI. (From Hily-Blant et al., in prep.).

(1996) to be a proxi of the vorticity, being the los average of the pos projection of the vorticity.

The most recent maps carried out at the IRAM-30m telescope in the ${ }^{12} \mathrm{CO}(2-1)$ line, produce up to $\sim 10^{5}$ independent spectra sampling homogeneous turbulence in diffuse molecular gas. An example is shown in Fig. 6. (Hily-Blant \& Falgarone, 2009, hereafter HF09, Hily-Blant et al., in prep.). The CVI-pdfs in that field have the anticipated nonGaussian wings that increase as the lag over which the increment is measured decreases (Fig. 7, left). The locus of the extreme CVIs (the E-CVIs) that build up the non-Gaussian 
wings of the $p d f \mathrm{~s}$ is an elongated narrow structure $(\sim 0.03 \mathrm{pc}$ thick $)$, almost straight over more than one parsec and surrounded by an ensemble of weaker, shorter structures (Fig. 7, right). As expected, the lane of largest E-CVIs coincides with the region where the velocity-shears are the largest, $40 \mathrm{~km} \mathrm{~s}^{-1} \mathrm{pc}^{-1}$ (see HF09). Most interestingly, it also coincides with a lane of weak ${ }^{12} \mathrm{CO}(2-1)$ emission and one of the weakest filaments of dust thermal emission detected at $250 \mu \mathrm{m}$ in that field with Herschel/SPIRE (MivilleDeschênes et al., 2010, Men'shchikov et al., 2010). Last, two low-mass dense cores in the field lie at the South-East tip of the E-CVIs locus (Heithausen et al., 2002), suggesting a causal link between bursts of turbulence dissipation, the formation of CO molecule, the formation of tenuous dense filaments and that of low-mass dense cores. The first of these steps is detailed in Section 5 .

A similar analysis has been performed in a cloud edge of the Perseus-Taurus-Auriga giant molecular complex. The field has the same total hydrogen column density as that in the Polaris Flare, but is less turbulent (half the pc-scale rms velocity dispersion). The CVI- $p d f$ s show departures from a Gaussian distribution with an amplitude 2.5 times smaller than in the Polaris Flare (Hily-Blant et al., 2008), in agreement with the theoretical predictions of Mininni et al. (2006).

This ensemble of properties, (i) the increasing departure of CVI- $p d f$ s from a Gaussian distribution as the scale decreases, (ii) the spatial coherent structures of E-CVIs and (iii) the link between the large-scale properties of turbulence and the magnitude of the smallscale E-CVIs, suggests that the ${ }^{12} \mathrm{CO}(2-1)$ E-CVIs trace the intermittency of turbulence in diffuse molecular clouds. The multifractal geometry of the E-CVIs structures in the Polaris Flare probed by the non-linear dependence of $\zeta_{p}$ with $p$ up to $p=6$ discussed in Hily-Blant et al. (2008) is now confirmed, up to higher orders, by the analysis of this larger data set. Unexpectedly, the $\zeta_{p}$ departure from a linear dependence agrees, within the error bars, with the She \& Levêque (1994) predictions for incompressible turbulence.

\subsection{Milliparsec-scale observations : approaching the dissipation scales}

A step further towards small-scales is provided by IRAM Plateau de Bure Interferometer (IRAM-PdBI) ${ }^{12} \mathrm{CO}(1-0)$ line observations of the field shown in Fig. 7 (right). A mosaic of 13 fields covers the $1^{\prime} \times 2$ ' area with a resolution of $\sim 4 \operatorname{arcsec}$ or $3 \mathrm{mpc}$ (Falgarone et al., 2009). These observations are unique so far : the lines detected are very weak, but the spatial dynamic range provided by the mosaic is large enough to allow the detection of 8 elongated structures with thickness as small as $\approx 3 \mathrm{mpc}(600 \mathrm{AU})$ and length up to $70 \mathrm{mpc}$, the size of the mosaic. Three are shown in Fig. 8 (left panels). They are not filaments because once merged with short-spacing data, the PdBI-structures appear to be the sharp edges of extended CO emission (Fig. 8, right panels). Moreover, six out of eight form pairs of quasi-parallel structures at different velocities. This cannot be due to chance alignment. Upper limits of the velocity-shears estimated for the three pairs (i.e. not corrected for projection effects) include the largest values ever measured in non-starforming regions, up to $780 \mathrm{~km} \mathrm{~s}^{-1} \mathrm{pc}^{-1}$ : two velocity components separated by $3.5 \mathrm{~km}$ $\mathrm{s}^{-1}$, a value of the order of the rms velocity dispersion of the CNM (Haud \& Kalberla 2007), share an interface as thin as $4.5 \mathrm{mpc}$ (in projection) with no shock signature nor density enhancement. Finally, the PdBI-structures are almost straight and their different position angles, $P A=60^{\circ}, 100^{\circ}$ and $165^{\circ}$, cover the same broad range of values as the projection of the magnetic field inferred from absorption towards field stars over much larger scales (Hily-Blant et al., in prep.). This suggests an interesting alignment of the pos projections of magnetic field and vorticity in these structures.

Falgarone et al. (2009) argue that, because the extended velocity components have sharp edges and because the small overlap of the pairs of velocity components cannot 

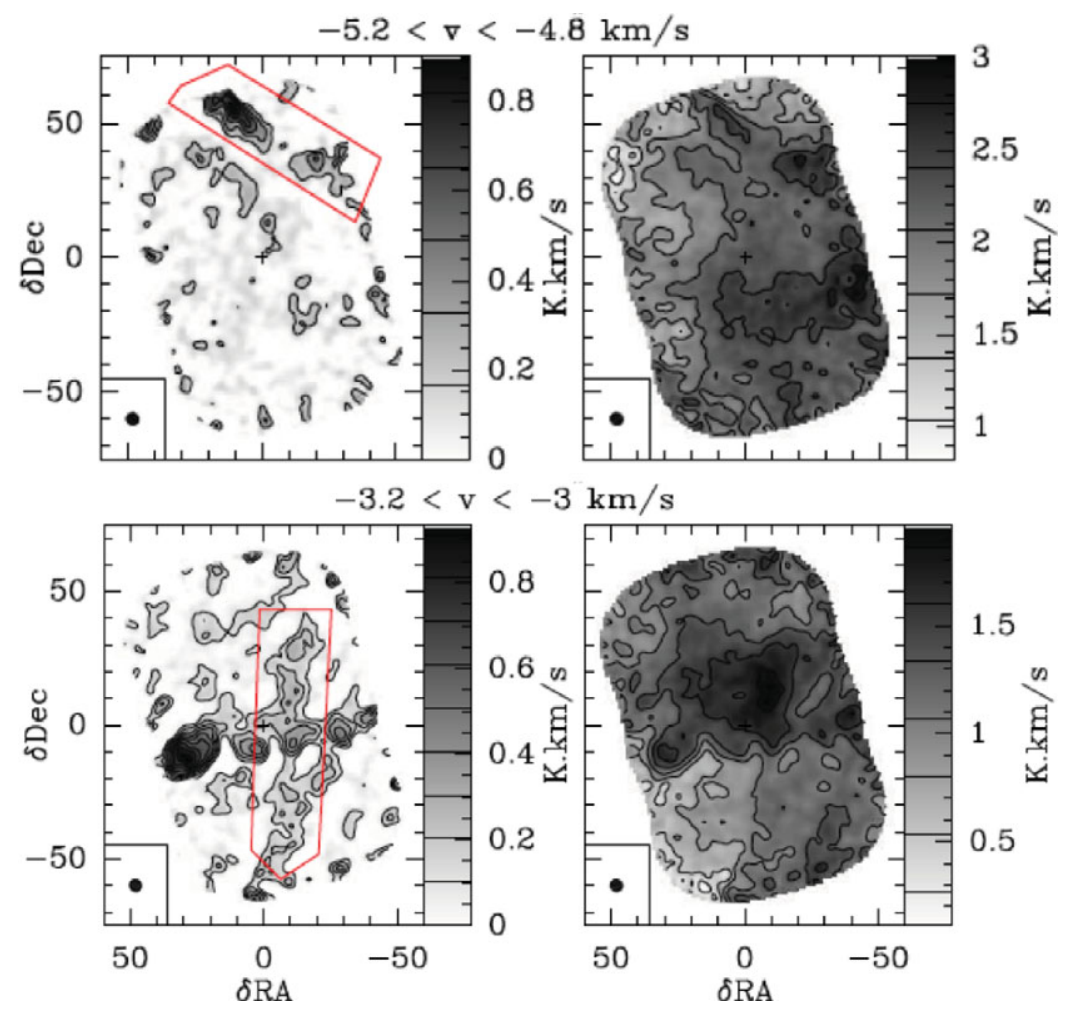

Figure 8. Three of the eight filamentary structures detected in the ${ }^{12} \mathrm{CO}(1-0)$ line with the IRAM-PdBI in the $2^{\prime} \times 1$ ' small field of the Polaris Flare drawn in Fig. 7. The synthesized beam $\sim 4 \operatorname{arcsec}\left(\right.$ or $3 \mathrm{mpc}$ ) is shown. Left panels: ${ }^{12} \mathrm{CO}(1-0)$ emission (integrated over the velocity range indicated above the panels) detected by the interferometer. Right panels: ${ }^{12} \mathrm{CO}(1-0)$ emission integrated over the same velocity intervals and including the short-spacings provided by the IRAM-30m telescope. The elongated structures seen by the interferometer appear as the sharp edges of weak extended CO structures. The structure in the top panel is coinciding with the $\sim 0.4$ pc long E-CVI structure that crosses the PdBI mosaic frame (Fig. 7).

be due to a chance orientation and has to occur for any viewing angle, these extended components have to be thin sheets of $\mathrm{CO}$ emission, not volumes. A similar reasoning applies to the geometry of the large velocity-shear detected in the single-dish data (HF09). The edges detected by the PdBI mark a discontinuity, at the milliparsec scale, between a detected CO-rich and an undetected CO-poor component (presumably the CNM), coinciding with a large velocity-shear. A low density $\left(\sim 50 \mathrm{~cm}^{-3}\right)$ CO-rich component would have been detected, although sub-thermally excited, because the sensitivity of the ${ }^{12} \mathrm{CO}(1-0)$ observations allows the detection of column densities as low $N(\mathrm{CO}) \sim 10^{14}$ $\mathrm{cm}^{-2}$ in velocity bins of $0.1 \mathrm{~km} \mathrm{~s}^{-1}$. These may be the first directly detected manifestations of the intermittency of interstellar turbulent dissipation. The large velocity-shears reveal an intense straining field, generating a local dissipation rate much larger than average, acting as a possible driver of CO formation (see Section 5).

Many surfaces in turbulent flows, in particular those of iso-dissipation, have been shown to be fractal with a dimension $D=2+\xi$ where $\xi$ is the scaling exponent of the velocity structure function ( $\xi=1 / 3$ in Kolmogorov turbulence) (Sreenivasan et al., 1989). If, as it seems to be the case, $\mathrm{CO}$ molecules form in the regions of intense turbulent dissipation in diffuse gas, it is not surprising that their spatial distribution be a fractal structure of dimension 1.3 in projection at the parsec scale. This result supports the conjecture 
linking the fractal distribution of $\mathrm{CO}$ emission in cloud edges to the power spectrum of turbulence made by Falgarone et al. (1991). Since, as expected from the behavior of $\zeta_{p}$ with $p$ (Hily-Blant et al., 2008), intermittency in the diffuse ISM is multi-fractal, the fractal dimension increases as the range of scales over which it is computed decreases, in agreement with the observed dependence of $\beta$ with length-scale (Falgarone et al., 2004).

These findings broaden the perspective on small-scale CO structures in diffuse molecular clouds. Isolated $\mathrm{CO}$ emission has been found in the high latitude sky (Heithausen, 2006) with bright sub-structures seen in PdBI observations (Heithausen, 2004). The substructures, although analysed as clumps by the author, are not randomly scattered in the field but form two elongated patterns, reminiscent for their thickness and length of what is found in FPH09, providing a velocity shear of $180 \mathrm{~km} \mathrm{~s}^{-1} \mathrm{pc}^{-1}$. Sakamoto \& Sunada(2003) have discovered a number of CO small-scale structures at the edges of the Taurus molecular cloud. Their main characteristics are their large line-width and their sudden appearance and disappearance within 0.03 to $0.1 \mathrm{pc}$. Interestingly, the authors propose that their small-scale CO structures pinpoint CO molecule-forming regions, driven by the thermal instability in the turbulent diffuse ISM. Finally, sensitive ${ }^{12} \mathrm{CO}(1-0)$ observations of the high-latitude sky by Barriault et al. (2010) show a good coincidence between the emergence of ${ }^{12} \mathrm{CO}(1-0)$ emission and $\mathrm{HI}$ velocity-shears.

Models of non-equilibrium chemistry in diffuse gas have attempted to capture the essence of the coupling between the physics of turbulent dissipation and the warm chemistry it triggers (Falgarone et al., 2005, Joulain et al., 1998, Godard et al., 2009). In the next Section, we summarize the most recent developments (Godard et al., in prep.).

\section{The predictions of the turbulent dissipation model}

\subsection{Outline of the model}

The TDR model, (for Turbulent Dissipation Regions, Godard et al., 2009, 2010) is based on the fact that turbulent dissipation that involves $\nabla \cdot \mathbf{v}$ and $\nabla \times \mathbf{v}$ is an intermittent quantity. The model relies on an analytical solution of the Helmholtz equation for the vorticity, the Burgers vortex. In addition to the traditional parameters of chemistry models, the density $n_{\mathrm{H}}$ and UV shielding characterized by the visual extinction $A_{V}$, the free parameters of the TDR model are constrained by the known large-scale properties of turbulence in the diffuse medium (see Joulain et al., 1998): the rate-of-strain $a-\mathrm{a}$ quantity homogeneous to $\mathrm{s}^{-1}-$ and the maximum orthoradial gas velocity in the vortex. The rate-of-strain and the equilibrium radius of the vortex are related by $r_{0}^{2}=4 \nu / a$. A hydrodynamical steady-state of a magnetized Burgers vortex of finite length is computed. The density is low enough that the neutral component decouples from the ions and magnetic field in the layers where the orthoradial velocity is the largest. The thermal and chemical evolution of a fluid cell crossing the steady-state vortex is computed at each time step, in a Lagrangian frame, with 127 species interacting via 2230 reactions. In this model, the dissipation is due to (1) viscous dissipation in the layers of intense velocity-shear at the vortex outer boundary and (2) ion-neutral friction induced by the decoupling of the ionized and neutral flows in the central regions.

Since the diffuse medium has a low density, its chemical and thermal inertia are large. The TDR model takes into account the long-lasting relaxation period that follows the end of any dissipative burst (the vortex lifetime i.e. the duration of the burst of dissipation, is set by energetic considerations, see Godard et al., 2009). A random line of sight through the medium therefore samples three kinds of gas : active vortices, relaxation phases and the ambient medium. The importance of the relaxation phase in the final column 

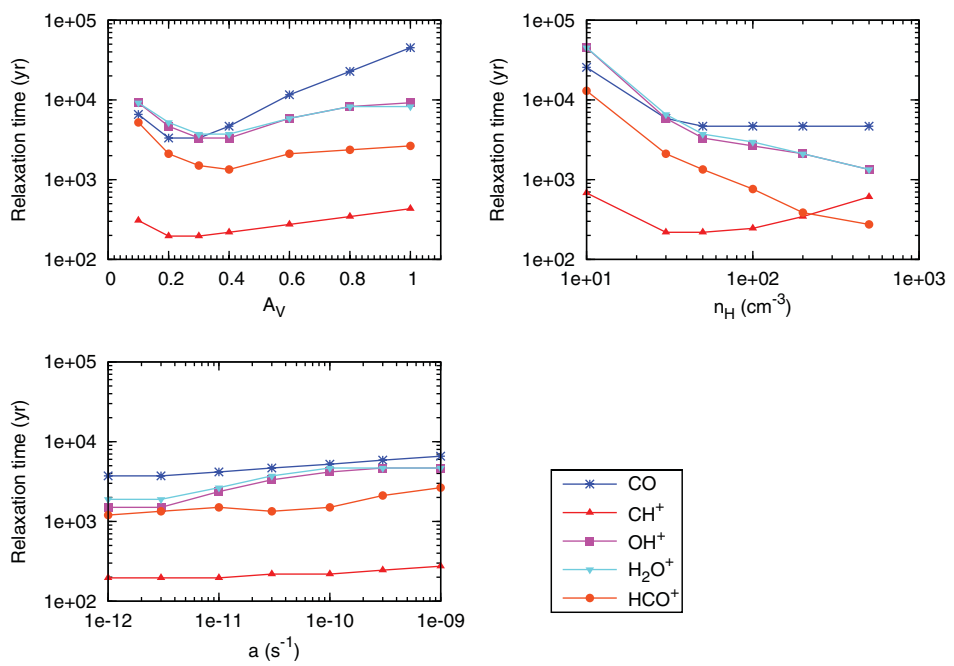

Figure 9. Relaxation times of different species as a function of the shielding from the ambient UV radiation field, $A_{V}$ (top left), the density, $n_{\mathrm{H}}$ (top right) and the turbulent rate-of-strain, a (bottom left). Note that these quantities are not model dependent.

density of a given species is proportional to its duration. The relaxation times of a subset of species are displayed in Fig. 9 : they cover a broad range, from $200 \mathrm{yr}$ for $\mathrm{CH}^{+}$ up to $5 \times 10^{4}$ yr for CO. Note that these large differences are model independent. This introduces a potentially untractable complexity in the comparison of the abundances of different species (see below). For instance, it is only at relatively high density that $\mathrm{CH}$ can be considered as a tracer of $\mathrm{H}_{2}$ in the ambient phase (Gerin et al., 2010). In all other cases, it is, as is $\mathrm{CH}^{+}$, a result of the warm chemistry.

\subsection{Scaling of $\mathrm{CH}^{+}$abundances with dissipation rate}

A large number of models have been run to explore the parameter space varying the density $\left(30<n_{\mathrm{H}}<500 \mathrm{~cm}^{-3}\right)$, the visual extinction $\left(0.1<A_{V}<2 \mathrm{mag}\right)$, the rateof-strain $\left(10^{-12}<a<10^{-10} \mathrm{~s}^{-1}\right)$ and the average turbulent energy transfer rate, $\epsilon$, up to 10 times its solar neighbourhood value (Falgarone et al., 2004). This quantity is assumed to be equal to the dissipation rate $\bar{\epsilon}_{D}$ averaged over time or space. The $\mathrm{CH}^{+}$ abundance is found to be proportional to $\bar{\epsilon}_{D}$, which makes this radical a specific tracer of the dissipation of suprathermal energy in the ISM. The scaling of $\mathrm{CH}^{+}$with $n_{\mathrm{H}}$ and $A_{V}$ is inferred from the model results:

$$
N\left(\mathrm{CH}^{+}\right) / N_{\mathrm{H}} \sim 2 \times 10^{-8}\left(\epsilon / 10^{-24} \mathrm{erg} \mathrm{cm}^{-3} \mathrm{~s}^{-1}\right)\left(n_{\mathrm{H}} / 50 \mathrm{~cm}^{-3}\right)^{-2.3}\left(A_{V} / 0.2\right)^{-1}
$$

Note the interesting increase of the $\mathrm{CH}^{+}$abundance as the UV-shielding decreases. This unique behavior follows from the destruction route of $\mathrm{CH}^{+}$due primarily to collisions with $\mathrm{H}$ and $\mathrm{H}_{2}$ rather than to photodissociation, with $\mathrm{CH}_{2}^{+}$and $\mathrm{CH}_{3}^{+}$being produced. Unlike $\mathrm{CH}^{+}$, these species are destroyed by UV photons, reforming $\mathrm{CH}^{+}$as a photodissociation product. This was found in MHD-shock models of DR21 (Falgarone et al., 2010a) where the behavior of $\mathrm{H}_{2} \mathrm{O}$ and $\mathrm{CH}^{+}$as the UV radiation field increases are found to be anticorrelated.

\section{3. $\mathrm{CH}^{+}, \mathrm{HCO}^{+}$and $\mathrm{CO}$, tracers of turbulent dissipation in the diffuse ISM}

In the warm chemistry network of the TDR model, $\mathrm{HCO}^{+}$is a daughter molecule of $\mathrm{CH}^{+}$, via the reaction $\mathrm{CH}_{3}^{+}+\mathrm{O} \rightarrow \mathrm{HCO}^{+}+\mathrm{H}_{2}$, while in the traditional UV-driven chemistry it is a daughter of $\mathrm{CO}^{+}$and water. $\mathrm{CO}$ in turn is produced by dissociative recombination of $\mathrm{HCO}^{+}$. The predicted column densities of $\mathrm{CH}^{+}$and $\mathrm{CO}$ are displayed in Fig. 10 as a function of that of $\mathrm{HCO}^{+}$for a broad range of densities, UV-shieldings 

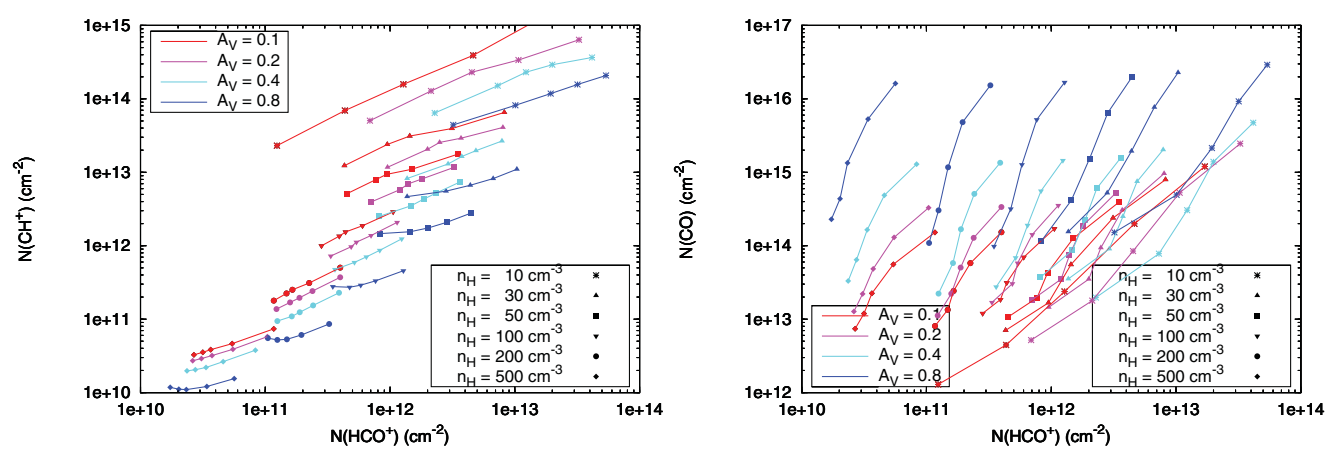

Figure 10. $\mathrm{CH}^{+}, \mathrm{HCO}^{+}$and $\mathrm{CO}$ column densities obtained in the TDR model per magnitude of gas sampled, for a range of proton densities and screenings from the UV field. The free parameter along each curve is the rate-of-strain ranging from $a=10^{-12} \mathrm{~s}^{-1}$ (upper right) to $a=10^{-10} \mathrm{~s}^{-1}$ (bottom left), where the model reaches the limits of its validity. The column densities decrease as $a$ increases because larger $a$ imply smaller vortex radii, therefore more dissipative vortices, but less numerous because the transfer rate of turbulent energy in the cascade is kept the same.

and rates-of-strain, and for a total gas column density of $N_{\mathrm{H}}=1.8 \times 10^{21} \mathrm{~cm}^{-2}$ or 1 mag.

While $\mathrm{CH}^{+}$and $\mathrm{HCO}^{+}$follow each other smoothly, the increase of $\mathrm{CO}$ with $\mathrm{HCO}^{+}$is much steeper as $a$ increases. This is because $\mathrm{CH}^{+}$(and $\mathrm{HCO}^{+}$to a lesser extent) does not survive beyond the end of the active phase of the vortex, while its long relaxation time (Fig. 9) makes $\mathrm{CO}$ observable for a much longer time after the vortex blast. It is remarkable that the column densities of $\mathrm{HCO}^{+}$observed in the diffuse medium, in the range of a few $10^{11}$ to $8 \times 10^{12} \mathrm{~cm}^{-2}$, are naturally reproduced by most models as soon as $\mathrm{CH}^{+}$is in the observed range of a few $10^{12}$ to $\sim 8 \times 10^{13} \mathrm{~cm}^{-2}$. Unfortunately, these two species have never been observed along the same los, but the above column densities all refer to low extinction los (i.e. total $\mathrm{H}$ column densities corresponding to $A_{V}=1$ mag, within a factor of a few) since $\mathrm{HCO}^{+}$is observed in the direction of QSOs. The CO and $\mathrm{HCO}^{+}$column densities observed by Liszt \& Lucas (1998) on the same los in the diffuse medium are bracketed by the model predictions for densities in the range 50-200 $\mathrm{cm}^{-3}$ and $A_{V}=0.4$ to $0.8 \mathrm{mag}$. The lowest $N(\mathrm{CO})$ observed in the diffuse ISM are those measured in absorption spectroscopy against nearby stars by Sonnentrucker et al. (2007) down to $N(\mathrm{CO}) \sim$ a few $10^{12} \mathrm{~cm}^{-2}$ for $N\left(\mathrm{H}_{2}\right)$ only a few $10^{19} \mathrm{~cm}^{-2}$.

The observed values tend to be in better agreement with low rates-of-strain i.e. models in which dissipation is dominated by ion-neutral friction. Remarkably, the pc-scale velocity shear of $40 \mathrm{~km} \mathrm{~s}^{-1} \mathrm{pc}^{-1}$ observed in the Polaris Flare would correspond to a straining field from the large scales $a=1.5 \times 10^{-12} \mathrm{~s}^{-1}$, while the intense small-scale shear of $780 \mathrm{~km} \mathrm{~s}^{-1} \mathrm{pc}^{-1}$ detected at the mpc-scale is 20 times larger $\left(a=3 \times 10^{-11}\right.$ $\mathrm{s}^{-1}$ ), comparable to the velocity-shear at the edge of the Burgers vortex for that rate-ofstrain. It is therefore possible that, alike $\mathrm{CH}^{+}$and $\mathrm{HCO}^{+}$, the $\mathrm{CO}$ emission detected in the diffuse molecular medium be a direct tracer of turbulent dissipation.

\section{4. $\mathrm{H}_{2}$ and $\mathrm{C}^{+}$radiative cooling rates}

The last interesting prediction of the TDR model is the intensity of the radiative cooling during the thermal relaxation phase (see also Falgarone et al., 2006). Fig. 11 displays the gas radiative cooling in the $\mathrm{H}_{2}$ pure rotational lines, the fine-structure [C II] and [O I] lines and the $\mathrm{CO}$ rotational lines as a function of the gas temperature (left) and time (right). While the $\mathrm{H}_{2}$ line emission is more intense than that of $\mathrm{C}^{+}$, it lasts only for the 

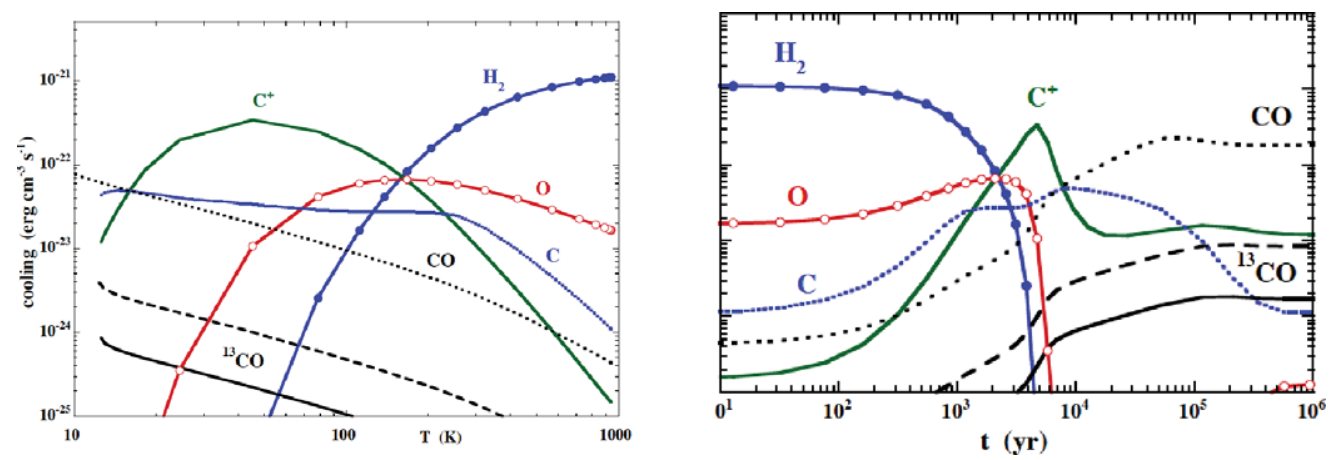

Figure 11. Radiative cooling rates of the pure rotational lines of $\mathrm{H}_{2}$, the fine structure line of $\mathrm{C}^{+}$and $\mathrm{OI}$ and the ${ }^{12} \mathrm{CO}$ and ${ }^{13} \mathrm{CO}$ rotational lines during the isobaric thermal relaxation of a burst of dissipation, left: as a function of gas temperature and right: as a function of time. The latter display shows that the total energy loss in the $\mathrm{H}_{2}$ and the $\mathrm{C}^{+}$lines are comparable.

short interval of time when the gas is warmer than $100 \mathrm{~K}$. Then, as the gas cools down, the $[\mathrm{C}$ II] line emission takes over. It is remarkable that the total energy radiated by these two species during the relaxation phase is about the same, the latter being about ten times weaker on average over a duration ten times larger. The $\mathrm{H}_{2}$ emission in the TDR model is comparable to that observed (Falgarone et al., 2005, Ingalls et al., in prep.), and the $[\mathrm{C}$ II] emission from a gas component heated by turbulent dissipation rather than by $\mathrm{UV}$ photons is predicted to be a possible significant fraction of the total $[\mathrm{C} \mathrm{II}]$ emission of the diffuse medium.

\section{Summary and perspectives}

The signatures of space-time intermittency of turbulence in diffuse molecular gas are clearly present. ${ }^{12} \mathrm{CO}(2-1)$ maps of large fields carried out at high angular resolution provide an unprecedented view of diffuse molecular gas and allow a powerful statistical analysis of the velocity field. In particular, the non-Gaussian statistics of the velocity field reveal parsec-scale coherent structures of intense velocity-shear with sub-structures observed down to $3 \mathrm{mpc}$. The large velocity-shears associated with the mpc-scale structures and their topology suggest that these are the possible birth sites of CO molecules in the diffuse medium.

Models of turbulent dissipation regions (TDR) in which non-equilibrium chemistry is triggered by intermittent bursts of dissipation in the diffuse ISM are encouraging. They involve slightly helical magnetic fields aligned with the local intense vorticity and partial decoupling of the ions and neutrals in short-lived dissipative structures filling, on average, only a few per cent of the diffuse medium. The abundances of almost all molecular species so far unexplained in the diffuse ISM, and its pure rotational $\mathrm{H}_{2}$ emission, are reasonably reproduced when a small fraction of active dissipative structures, and relaxation phases are considered in the los modelling. These models are the first attempt to bridge the chemistry to the huge range of scales coupled by the turbulent cascade, and do so in a theoretical framework. They provide relevant boundary conditions for the chemistry models, by providing a way to take into account, in a self-consistent way, the amount of supra-thermal energy that is injected at the mpc-scale, and the associated timescales. They have their limitations, though: the analytical solution of the Burgers vortex itself, the lack of retro-action of the gas chemistry (and therefore cooling, and ionization degree) 
on the dynamical (hydro and magnetic) steady-state, the los modelling ignoring any distribution of vortex properties, to name a few.

On the observational side, the nature of the dissipative structures (shocks or pure velocity-shears), the actual size of the dissipation scale, the fractal geometry of the smallest scales, and the topology of the magnetic field in these structures are open fields for future observations. ALMA should allow observations of the diffuse ISM between 1000 $\mathrm{AU}$ and $10 \mathrm{AU}$. On the theoretical side, the pictured evolution from coherent structures of intense velocity-shears to molecule formation, enhanced radiative cooling, gas condensation and formation of a network of dense gas filaments, is still to be computed.

\section{References}

de Avillez, M. A. \& Breitschweirdt D. 2005 A $\mathscr{E} A 436585$

Anselmet, F., Antonia, R. A., \& Danaila, L. 2001, Planetary E Space Sciences, 49, 1177

Audit, E. \& Hennebelle, P. 2005, A\& A, 433, 1

Barriault, L., Joncas, G., Falgarone, E. et al. 2010 MNRAS 4062713

Begum, A., Stanimirovic, S., Goss, M., Heiles, C., Pavkovich, A., \& Hennebelle, P. 2010 ApJ 7251779

Cox, D. 2005, ARAA 43, 337

Crane, P., Lambert, D. L., \& Sheffer, Y. 1995 ApJS 99, 107

Crutcher, R., Wandelt, B., Heiles, C., Falgarone, E., \& Troland, T. 2010, ApJ, 725, 466

Elmegreen, B. G. \& Scalo, J. 2004, ARAA, 42, 211

Falgarone, E., Phillips, T. G., \& Walker, C. K. 1991, ApJ, 378, 186

Falgarone, E., Hily-Blant, P., \& Levrier, F., 2004, Ap\&SSS, 292, 89

Falgarone, E., Verstraete, L., Pineau des Forêts, G., \& Hily-Blant, P. 2005, A\&A, 433, 997

Falgarone, E., Pineau des Forêts, G., Hily-Blant, P., \& Schilke, P. 2006, A $\& A$, 452, 511

Falgarone, E., Pety, J., \& Hily-Blant, P. 2009, A\&A, 507, 355

Falgarone, E., Ossenkopf, V., Gerin, M., et al., 2010a, A\&A 518, L118

Falgarone, E., Godard, B., Cernicharo, J. et al., 2010b, A\& $A$ 521, L15

Federrath, C., Duval, J., Klessen, R. S., et al., 2010, A\&A 512, 81

Field, G., Goldsmith, D., \& Habing, H. 1969 ApJ 155, L149

Fitzpatrick, E. L. \& Spitzer, L. 1997, ApJ, 745, 623

Frisch, U. 1995, Turbulence : the legacy of A.N. Kolmogorov, Cambridge University Press, Cambridge

Gerin, M., de Luca, M., Goicoechea, J. et al., 2010 A\&A 521, L16

Godard, B., Falgarone, E., \& Pineau des Forêts, G. 2009, A $\xi A, 495,847$

Godard, B., Falgarone, E., Gerin, M. Hily-Blant, P., \& de Luca, M., 2010, A\& A, 520, A20

Godard, B., Falgarone, E., Gerin, M., et al., 2011, submitted to $A \mathscr{\mho} A$

Goldsmith, P. F., Heyer, M., Narayanan, G., et al., 2008, ApJ, 680, 428

Goldsmith, P. F., Velusamy, T., Li, D., \& Langer W. D. 2010, ApJ, 715, 1370

Gredel, R., 1997 A $\dot{E}$ 320, 929

Hartmann J. 1904 ApJ 19, 268

Haud, U. \& Kalberla, P. M. W. 2007, A $\mathscr{E} A, 466,555$

Heiles, C. \& Troland, T. 2003 ApJ 5861067

Heithausen, A. 2004, ApJ, 606, L13

Heithausen, A. 2006, A\& $A, 450,193$

Heithausen, A., Bertoldi, F., \& Bensch, F. 2002, A\& $A$, 383, 591

Hewitt J. W., Rho J., Andersen, M., \& Reach W. T. 2009 ApJ 694, 1266

Hily-Blant, P. \& Falgarone, E. 2007, A\&A, 469, 173

Hily-Blant, P., Falgarone, E., \& Pety, J. 2008, A\& $A$, 481, 367

Hily-Blant, P. \& Falgarone, E., 2009, A\&A, 500, L29 (HF09)

Jenkins, E. B. \& Tripp, T. M. 2011, ApJ, 734, 65

Joulain, K., Falgarone, E., Pineau des Forêts, G. P., \& Flower, D. 1998, A\&\&A, 340, 241 
Kainulainen, J., Beuther, H., Henning, T., \& Plume, R. 2009 A $\& A$ 508,L35

Kolmogorov, A. N. 1941, Dokl. Akad. Nauk SSSR, 26, 115

Kolmogorov, A. N. 1962, J. Fluid Mech., 13, 82

Landau, L. E. \& Lifchitz, E. M. 1959, Fluid Mechanics, Pergamon Press, Oxford

Lis, D. C., Pety, J., Phillips, T. G., \& Falgarone, E. 1996, ApJ, 463, 623

Liszt, H. S. \& Lucas, R. 1998, A\&A, 339, 561

Mac Low, M.-M., Klessen, R. S., Burkert, A., \& Smith, M. D. 1998, Phys. Rev. Lett., 80, 2754

Menten, K., Wyrowski, F., Belloche, A., et al., 2011 A\&A 525, 77

Men'shchikov, A., André, P., Didelon, P., et al., 2010 A $\& A 518$ L103

Mininni, P. D., Alexakis, A., \& Pouquet, A. 2006, Phys. Rev. E, 74, 6303

Miville-Deschênes, M.-A., Joncas G., Falgarone, E., \& Boulanger, F., 2003, A\&A, 411, 109

Miville-Deschênes, M.-A., Martin P., Abergel A., et al., 2010, A\&̈A, 518 L104

Miville-Deschênes, M.-A. \& Lagache, 2005, ApJS 157, 302

Moffatt, H. K., Kida, S., \& Ohkitani, K. 1994, J. Fluid Mech., 259, 241

Moisy, F. \& Jiménez, J. 2004, J. Fluid Mech., 513, 111

Pety, J. \& Falgarone, E. 2003, A $\& A, 412,417$

Politano, H. \& Pouquet, A. 1995 Phys. Rev. E, 52, 636

Porter, D. H., Pouquet, A., \& Woodward, P. R. 2002, Phys. Rev. E, 66, 026301

Sakamoto, S. \& Sunada, K. 2003, ApJ, 594, 340

Scalo, J. \& Elmegreen, B. G. 2004, ARAA, 42, 275

She, Z.-S. \& Levêque, E. 1994, Phys. Rev. Lett. 72, 336

Snow, T. P. \& McCall, B. J. 2006, ARAA, 44, 367

Sonnentrucker, P., Welty, D. E., Thorburn, J. A. \& York, D. G. 2007 ApJS 16858

Sreenivasan, K. R., Ramshankar, R., \& Méneveau, C. 1989, Proc. R. Soc. Lond. A, 421, 79

Vestuto, J. G., Ostriker, E. C., \& Stone, J. M. 2003, ApJ, 590, 858

Weselak, T., Galazutdinov, G., Musaev, F. \& Krelowski, J., 2008 A\&\&A 479149

\section{Discussion}

AIKAWA: What is the initial conditions of the chemistry model? Is the criterion of $A_{V} \sim$ 0.2 set by PDR models?

FALGARONE: The initial conditions of the chemistry are set by PDR models. The $\mathrm{H}_{2}$ fraction at the low shielding value $A_{V} \sim 0.2$ is therefore very low.

Ossenkopf: In a number of sources, we see $\mathrm{CH}^{+}$with very narrow linewidths of a few $\mathrm{km} \mathrm{s}^{-1}$. Isn't this a contradiction with the large-shear production?

FALGARONE: Such linewidths are not narrow, strictly speaking. They are comparable to those found in visible absorption lines sampling local diffuse gas and to the width of individual gas velocity components in submillimeter lineprofiles across the galactic diffuse medium. However, there is not one single process involved in the formation of $\mathrm{CH}^{+}$. High velocity shocks produce $\mathrm{CH}^{+}$broad wings of several $10 \mathrm{~km} \mathrm{~s}^{-1}$ (e.g. Falgarone et al. 2010). In dense highly FUV illuminated PDRs (I do not know if these are the sources you refer to), the production of $\mathrm{CH}^{+}$is driven by reaction of $\mathrm{C}^{+}$with vibrationally excited $\mathrm{H}_{2}$ excited by fluorescence (Agundez et al., 2010). The supra-thermal energy being due to FUV photons, in that case, the $\mathrm{CH}^{+}$linewidths should not be any broader than those of other molecules. This mechanism is not efficient enough in diffuse clouds illuminated by the ambient interstellar radiation field (ISRF). The role of turbulent dissipation (and large velocity-shears) is dominant there. My talk was restricted to the diffuse medium irradiated by ISRF. 